\title{
Fractional topological phase for entangled qudits
}

\author{
L. E. Oxman and A. Z. Khoury \\ Instituto de Fúsica, Universidade Federal Fluminense, 24210-346 Niterói - RJ, Brasil.
}

\begin{abstract}
We investigate the topological structure of entangled qudits under unitary local operations. Different sectors are identified in the evolution, and their geometrical and topological aspects are analyzed. The geometric phase is explicitly calculated in terms of the concurrence. As a main result, we predict a fractional topological phase for cyclic evolutions in the multiply connected space of maximally entangled states.
\end{abstract}

PACS numbers: PACS: 03.65.Vf, 03.67.Mn, 07.60.Ly, 42.50.Dv

In a seminal work, M. Berry [1] showed the important role played by geometric phases in quantum theory. Since then, the interest for geometric phases was renewed by potential applications to quantum computation. The experimental demonstration of a conditional phase gate was provided both in Nuclear Magnetic Resonance (NMR) 2] and trapped ions [3]. Optical geometric phases have already been discussed both for polarization [4] and vortex mode transformations [5, 6]. The role of entanglement in the phase evolution of qubits was investigated in refs. [7, 8]. Recently, P. Milman and R. Mosseri [9, 10] investigated the geometric phase and the topological structure associated with cyclic evolutions of arbitrary two-qubit pure states. This structure has been experimentally evidenced in the context of spin-orbit mode transformations of a laser beam [11] and in NMR [12]. Although the topological nature of the phase acquired by maximally entangled states is well settled, the distinction between geometrical and topological phases has not been established clearly for partially entangled states. In this work we present a group theoretical approach which allows for a clear distinction between the two aspects. As a bonus, this approach is easily extended to higher dimensions, bringing an interesting prediction of a fractional topological phase.

Let $|\psi\rangle=\sum_{i, j=1}^{d} \alpha_{i j}|i j\rangle$ be the most general twoqudit pure state. We shall represent this state by the $d \times d$ matrix $\alpha$ whose elements are the coefficients $\alpha_{i j}$. With this notation the norm of the state vector becomes $\langle\psi \mid \psi\rangle=\operatorname{Tr}\left(\alpha^{\dagger} \alpha\right)=1$ and the scalar product between two states is $\langle\phi \mid \psi\rangle=\operatorname{Tr}\left(\beta^{\dagger} \alpha\right)$, where $\beta$ is the $d \times d$ matrix containing the coefficients of state $|\phi\rangle$ in the chosen basis. We are interested in the phase evolution of the state $|\psi\rangle$ under local unitary operations. So let us take two unitary matrices $U_{A}$ and $U_{B}$ belonging to $U(d)$ and representing the operations performed in each subsystem separately. Under these unitary operations the state matrix will evolve as $\alpha(t)=U_{A} \alpha(0) U_{B}^{\top}$, where $U_{j}(t)=e^{i \phi_{j}(t)} \bar{U}_{j}(t)(j=A, B)$ and $\bar{U}_{j} \in S U(d)$. One can identify the following invariants under local unitary evolutions: $\operatorname{Tr}\left[\rho_{j}^{p}\right], p=1, \ldots, d$, where $\rho_{j}$ is the reduced density matrix with respect to qudit $j\left(\rho_{A}=\alpha^{\top} \alpha^{*}\right.$ and $\left.\rho_{B}=\alpha \alpha^{\dagger}\right)$. In fact, the invariants are $j$-independent.
The first one $(p=1)$ is simply the norm of the state vector. One can readily relate the second invariant to the I-concurrence of a two-qudit pure quantum state 13] $\mathcal{C}=\sqrt{2\left(1-\operatorname{Tr}\left[\rho^{2}\right]\right)}$, so that its invariance expresses the well known fact that entanglement is not affected by local unitary operations. The $p=d$ invariant can be rewritten in terms of the former and $\mathcal{D}=|\operatorname{det}[\alpha]|$. In particular, for qubits we have $\mathcal{C}=2 \mathcal{D}$.

In the case of a cyclic evolution, $\bar{U}_{A}(\tau) \alpha(0) \bar{U}_{B}^{\top}(\tau)=$ $e^{i \Delta \phi} \alpha(0)$. By taking the determinant of both sides we get: $e^{i d \Delta \phi}=1$ as long as $\mathcal{D} \neq 0$. This implies that the possible acquired phases due to the $S U(d)$ part of a cyclic evolution are $\Delta \phi=2 \pi n / d$, with $n=0,1,2, \ldots, d-1$. For qubits $(d=2)$ one recovers the well known result $\Delta \phi=0, \pi$. However, for $d>2$ one obtains fractional phase values in steps of $2 \pi / d$. Now, we are interested in discussing in what sense this fractional phase can be considered as topological. For this aim, we will analyze the topology of the space of two-qudit states and how the total phase is built. In this regard, we would like to underline that according to ref. [14], the geometric phase acquired by a time evolving quantum state $\alpha(t)$ is always defined as

$$
\phi_{g}=\arg \langle\psi(0) \mid \psi(t)\rangle+i \int d t\langle\psi(t) \mid \dot{\psi}(t)\rangle,
$$

that corresponds to the total phase minus the dynamical phase. Therefore, a topological phase, that is, an object that only depends on a given class of paths, can only find room as a part of the geometric phase, an object that is invariant under reparametrizations and gauge transformations. Gauge invariance corresponds to the fact that the phase factors $\phi_{j}(t)$ do not contribute to $\phi_{g}$, which is completely determined by $\bar{U}_{j}(t)$, the sector where the fractional values occur.

In order to characterize the space of states, we note that any invertible matrix admits a polar decomposition $\alpha=Q S$, where $Q=\sqrt[d]{\mathcal{D}} e^{M}$ is a positive definite Hermitian matrix, $M$ is a traceless Hermitian matrix, and $S=e^{i \phi} \bar{S}, \bar{S} \in S U(d)$. Since $\operatorname{det}\left[e^{M}\right]=e^{\operatorname{Tr}[M]}=1$, one easily finds $\operatorname{det}(\alpha)=\mathcal{D} e^{i d \phi}$. We can identify the time evolution as occurring in different sectors

$$
\alpha(t)=\sqrt[d]{\mathcal{D}} e^{i \phi(t)} e^{M(t)} \bar{S}(t),
$$


where we have denoted, $\phi(t)=\phi(0)+\phi_{A}(t)+\phi_{B}(t)$, $M(t)=\bar{U}_{A}(t) M(0) \bar{U}_{A}(t)^{\dagger}$, and $\bar{S}(t)=\bar{U}_{A}(t) \bar{S}(0) \bar{U}_{B}^{\top}(t)$. Therefore, we identify the evolution in three sectors of the matrix structure: an explicit phase evolution $\phi(t)$, an evolution closed in the space of traceless Hermitian matrices $M(t)$, and the evolution $\bar{S}(t)$ closed in $S U(d)$.

Now we are able to discuss the topological aspects of the entangled state evolution in terms of these sectors. The space of positive definite Hermitian matrices $Q$ has trivial topology. This is a noncompact manifold isomorphous to $R^{d^{2}-1}$, as it can be parametrized in the form $Q=e^{\beta_{a} T_{a}}$, where $\beta_{a}$ are real numbers and $T_{a}$ $\left(a=1,2, \ldots, d^{2}-1\right)$ is a basis in the space of Hermitian traceless matrices. These $T_{a}$ 's are the generators of $S U(d)$, so that $\bar{S}=e^{i \omega_{a} T_{a}}$, with $\omega_{a}$ real. They can be normalized in the form $\operatorname{tr}\left(T^{a} T^{b}\right)=\frac{1}{2} \delta^{a b}$ and obey the Lie algebra $\left[T^{a}, T^{b}\right]=i f^{a b c} T^{c}$, where $f^{a b c}$ are the structure constants of $S U(d)$. The first homotopy group of $S U(d)$ is also trivial, however, the physical equivalence of $\alpha$ matrices differing by a global phase corresponds to considering the identification in $S U(d), e^{i 2 \pi n / d} \bar{S} \equiv \bar{S}$. This can be naturally implemented by associating the $S U(d)$ sector of the matrix $\alpha$ with a corresponding sector for the quantum states, represented by transformations $R(\bar{S})$ in the adjoint representation $\bar{S} T^{a} \bar{S}^{-1}=\hat{n}_{a} \cdot \vec{T}$, $\hat{n}_{a}=R(\bar{S}) \hat{e}_{a}$. In this manner, the matrices $e^{i 2 \pi n / d} \bar{S}$ are mapped to the same point $R(\bar{S})$. In other words, a part of the evolution can be parametrized as $R(t) \in \operatorname{Adj}(d)$, or equivalently, in terms of a time dependent frame $\hat{n}_{a}(t)$. Note that for qubits the adjoint representation corresponds to $S O(3)$, the manifold used in ref. [9, 10] to describe maximally entangled states. An evolution $\bar{S}(t)$ starting at $\bar{S}(0)$ and ending at $e^{i 2 \pi / d} \bar{S}(0)$ defines an open path in $S U(d)$ and a topologically nontrivial closed path $R(t) \in \operatorname{Adj}(d)$. If this cyclic evolution were composed $d$ times, we would get a trivial path in $\operatorname{Adj}(d)$, so that the number of nonequivalent classes is given by $d$.

The total phase can be written as

$$
\begin{aligned}
\phi_{\text {tot }}= & \arg \left\{\operatorname{Tr}\left[\alpha^{\dagger}(0) \alpha(t)\right]\right\}=\phi_{A}+\phi_{B} \\
& +\arg \left\{\operatorname{Tr}\left[\alpha^{\dagger}(0) \bar{U}_{A}(t) \alpha(0) \bar{U}_{B}^{\top}(t)\right]\right\},
\end{aligned}
$$

while the dynamical phase is,

$$
\begin{gathered}
\phi_{d y n}=-i \int_{0}^{t} d t^{\prime} \operatorname{Tr}\left[\alpha^{\dagger}\left(t^{\prime}\right) \dot{\alpha}\left(t^{\prime}\right)\right]=\phi_{A}+\phi_{B} \\
-i \int_{0}^{t} d t^{\prime} \operatorname{Tr}\left[\rho_{B}(0) \bar{U}_{A}^{\dagger} \dot{\bar{U}}_{A}+\rho_{A}^{\top}(0) \dot{\bar{U}}_{B}^{\top} \bar{U}_{B}^{*}\right],
\end{gathered}
$$

where $\rho_{A}=\left(S^{\dagger} Q^{2} S\right)^{*}, \rho_{B}=Q^{2}$. For cyclic evolutions we have $\bar{U}_{j}(\tau)=e^{i 2 \pi n_{j} / d} \bar{U}_{j}(0)$. Then, the total generated phase is $\phi_{t o t}=\phi_{A}+\phi_{B}+2 \pi n / d, n=n_{A}+n_{B}$, where the values $n \neq 0, d, 2 d, \ldots$, correspond to topologically nontrivial paths. As already discussed, the total phase is always written as a dynamical plus a geometric part. In order to consider a fractional phase as topological, it must be built only as a part of the geometric phase, receiving no relevant contribution from the dynamical part. This means that at any time $t, 0 \leq t \leq \tau$, we must have,

$$
\int_{0}^{t} d t^{\prime} \operatorname{Tr}\left[\rho_{B}(0) \bar{U}_{A}^{\dagger} \dot{\bar{U}}_{A}+\rho_{A}^{\top}(0) \dot{\bar{U}}_{B}^{\top} \bar{U}_{B}^{*}\right]=0 .
$$

This is satisfied by the maximally entangled states, for every possible local evolution $\bar{U}_{j}$. In this regard, the invariant quantities in the evolution can be written as $\operatorname{Tr}\left[\left(Q^{2}\right)^{p}\right]$. In terms of the concurrence we can write

$$
Q^{2}=(1 / d) I+\sqrt{\mathcal{C}_{m}^{2}-\mathcal{C}^{2}} \hat{q} \cdot \vec{T},
$$

where $\mathcal{C}_{m}=\sqrt{2(d-1) / d}$. The $\mathcal{C}=0$ value corresponds to separable states. For maximally entangled states $\mathcal{C}=\mathcal{C}_{m}$, giving $Q^{2}=(1 / d) I$, and $\rho_{A}=\rho_{B}=(1 / d) I$. In addition, for any $\bar{U}_{j} \in S U(d)$, the matrices $\bar{U}_{j}^{\dagger} \overline{\bar{U}}_{j}$ are combinations of the generators $T_{a}$. Therefore, using this information, the trace in the integrand of eq. (5) vanishes.

Now, let us consider an evolution on the first qudit $A$. In this case, $\langle\psi(0) \mid \psi(t)\rangle=\operatorname{Tr}\left[Q^{2}(0) \bar{U}_{A}(t)\right]$, while the dynamical phase is,

$$
\phi_{d y n}=\phi_{A}-i \int_{0}^{t} d t^{\prime} \operatorname{Tr}\left[Q^{2}(0) \bar{U}_{A}^{\dagger}\left(t^{\prime}\right) \dot{\bar{U}}_{A}\left(t^{\prime}\right)\right] .
$$

These phases do not depend on $\bar{S}(0)$ so that for simplicity we can consider $\bar{S}(0)=I$, that is, $\bar{U}_{A}(t)=\bar{S}(t)$. For qubits $T_{a}=\sigma_{a} / 2(a=1,2,3)$, where $\sigma_{a}$ are the Pauli matrices. We shall assume that the basis is chosen so that $\hat{q}(0)=\hat{e}_{3}$, that is, $Q^{2}=I / 2+\sqrt{1-\mathcal{C}^{2}} \sigma_{3} / 2$. The unitary sector of the state evolution can be put in terms of Euler angles, in the form $\bar{U}_{A}(t)=U_{m}(t) V_{3}(t)$, where

$$
U_{m}=e^{-i \varphi T_{3}} e^{i \theta T_{2}} e^{i \varphi T_{3}} \quad, \quad V_{3}=e^{i \chi T_{3}} .
$$

Note that for cyclic evolutions, $U_{m}(0)=U_{m}(\tau)$, while $V_{3}(0)= \pm V_{3}(\tau)$. In addition, $U_{m}$ can be expanded in terms of $I, T_{1}$ and $T_{2}$, as the term proportional to $T_{3}$ is obtained from $\operatorname{Tr}\left[T_{3} e^{-i \varphi T_{3}} e^{i \theta T_{2}} e^{i \varphi T_{3}}\right]=\operatorname{Tr}\left[T_{3} e^{i \theta T_{2}}\right]=$ 0 . Here, we have used that the latter exponential is a combination of $I$ and $T_{2}$. Using a similar expansion for $e^{i \varphi T_{3}}$, we arrive to the conclusion that the terms in $U_{m}$ proportional to $T_{1}, T_{2}$ do not contribute to $\langle\psi(0) \mid \psi(t)\rangle$.

With the ingredients above we can work out the expression for the time evolving overlap

$$
\langle\psi(0) \mid \psi(t)\rangle=e^{i \phi_{A}} \cos \frac{\theta}{2}\left[\cos \frac{\chi}{2}+i \sqrt{1-\mathcal{C}^{2}} \sin \frac{\chi}{2}\right] .
$$

In terms of $Q^{2}, U_{m}$, and $V_{3}$, the dynamical phase is

$$
\begin{aligned}
\phi_{d y n} & =\phi_{A}-i \int_{0}^{t} d t^{\prime} \frac{1}{2} \operatorname{Tr}\left[\left(I+\sqrt{1-\mathcal{C}^{2}} \sigma_{3}\right)\right. \\
& \left.\times\left(U_{m}^{\dagger} \dot{U}_{m}+V_{3}^{\dagger} \dot{V}_{3}\right)\right] .
\end{aligned}
$$




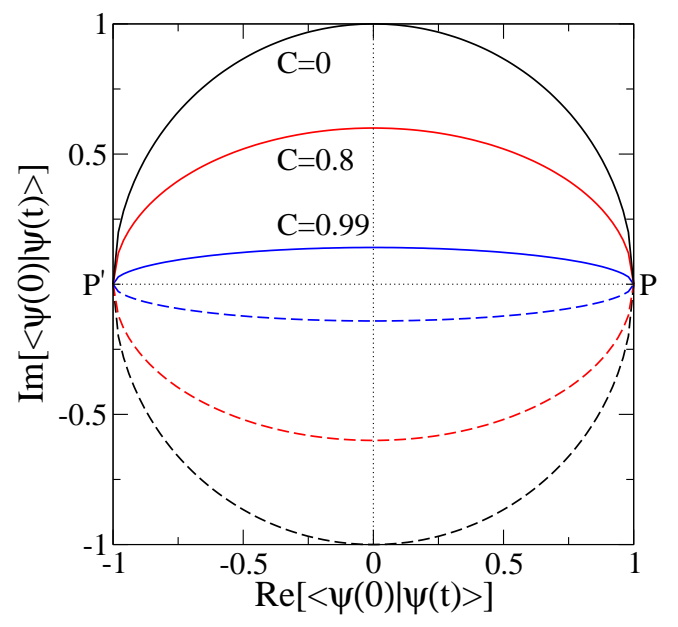

FIG. 1. Time evolution of the quantum state overlap for a pair of qubits with different concurrences.

Using $\dot{V}_{3}=i(\dot{\chi} / 2) \sigma_{3} V_{3}$ and defining the unit vectors $\hat{m}_{a}$ so that $U_{m} \sigma_{a} U_{m}^{\dagger}=\hat{m}_{a} \cdot \vec{\sigma}$, we get

$$
\begin{aligned}
\phi_{g} & =\arctan \left[\sqrt{1-\mathcal{C}^{2}} \tan (\chi / 2)\right]-\sqrt{1-\mathcal{C}^{2}}(\chi / 2) \\
& +\sqrt{1-\mathcal{C}^{2}}(\Phi / 2),
\end{aligned}
$$

with $\Phi \equiv \int_{0}^{t} d t^{\prime} \hat{m}_{1} \cdot \dot{\hat{m}}_{2}$. In the last term, the frame $\hat{m}_{a}$ depends on $\theta \in[0, \pi)$ and $\varphi \in[0,2 \pi]$ defining a point on $S^{2}$, the surface of a sphere with unit radius. Then, $\hat{m}_{a}(\theta, \varphi)$ is a mapping $S^{2} \rightarrow \hat{m}_{a}$, and the evolution on this sector is given by a curve, defined by $\theta(t), \varphi(t)$, contained on $S^{2}$. In this regard, for a cyclic evolution, one easily shows that $\Phi=\Omega$, where $\Omega$ is the solid angle subtended by the closed path [15, 16]. This term can be associated to the usual Berry phase for a single qubit.

For a general evolution, we see that for product states $(\mathcal{C}=0)$, the first two terms in eq. (11) cancel each other while the last term coincides with the one given by the usual picture of the Bloch sphere evolution of a single qubit. On the other hand, for maximally entangled states $(\mathcal{C}=1)$, the last two terms vanish while the first term can assume only two discrete values 0 or $\pi$. In fig 1 this evolution is represented as paths in the complex plane, where the overlap $\langle\psi(0) \mid \psi(t)\rangle$ is plotted for different values of the concurrence. This path degenerates to a circle for product states and to a straight line on the real axis as the concurrence approaches its maximum value $C=1$. It gives a graphical picture of the phase jump between 0 and $\pi$ discussed in ref. [10]. This jump occurs when the evolving state crosses the subspace orthogonal to the initial one. Note that the solid lines in fig 1 correspond to closed paths since points $P$ and $P^{\prime}$ represent physically equivalent quantum states. Dashed lines correspond to additional closed paths.

Now, let us study a simple nontrivial path that generalizes the $V_{3}$-sector for qubits (cf. eq. (8) ) to the case of qudits. Consider an evolution of the form $\bar{U}_{A}(t)=V_{N}(t)$,

$$
V_{N}(t)=e^{i \chi(t) E} \quad, \quad \chi(0)=0,
$$

where $E$ is a diagonal traceless matrix with components,

$$
E_{\alpha \alpha}= \begin{cases}(1 / d), & \alpha=1, \ldots, d-1 \\ (1 / d)-1, & \alpha=d .\end{cases}
$$

This matrix can be written in terms of the $N$ 'th generator of $S U(d), N=d^{2}-1: E=\mathcal{C}_{m} T_{N}$. When $\chi(\tau)=2 \pi$, it is simple to see that $V_{N}(\tau)=e^{i 2 \pi / d} I$. In the case where $\hat{q}(0)=\hat{e}_{N}$, we have,

$$
Q^{2}(0)=(1 / d) I+\sqrt{1-\left(\mathcal{C} / \mathcal{C}_{m}\right)^{2}} E .
$$

By expanding the exponential in eq. (12) and using eq.(14) we get,

$$
\langle\psi(0) \mid \psi(t)\rangle=\mathcal{A} e^{i \chi / d}+\mathcal{B} e^{i(1-d) \chi / d},
$$

with $\mathcal{A}=\frac{d-1}{d}+\frac{1}{2} \sqrt{\mathcal{C}_{m}^{2}-\mathcal{C}^{2}}$ and $\mathcal{B}=1-\mathcal{A}$. Using $V_{N}^{\dagger} \dot{V}_{N}=i \dot{\chi} E$ in the dynamical phase, we arrive at

$$
\phi_{g}=\arctan \left[\frac{\mathcal{A} \sin \frac{\chi}{d}+\mathcal{B} \sin \frac{(1-d) \chi}{d}}{\mathcal{A} \cos \frac{\chi}{d}+\mathcal{B} \cos \frac{(1-d) \chi}{d}}\right]-\sqrt{\mathcal{C}_{m}^{2}-\mathcal{C}^{2}} \frac{\chi}{2} .
$$

In the above example, for maximally entangled states, when $d \geq 3$ the total phase changes continuously from 0 to $2 \pi / d$, and the evolving state never becomes orthogonal to the initial state. This is in contrast to what happens in the $d=2$ case. The minimum value for $|\langle\psi(0) \mid \psi(t)\rangle|^{2}$ is $(\mathcal{A}-\mathcal{B})^{2}=\left(\frac{d-2}{d}\right)^{2}$, attained when $\chi=\pi$. For $d=3$, the minimum overlap is $(1 / 3)^{2}$.

It is interesting to look for topologically nontrivial evolutions for qudits with similar properties to those displayed by qubits. In the $d=3$ case, this can be realized as follows. Let us consider the path $\bar{U}_{A}(\chi(t))$, continuously evolving from $\bar{U}_{A}(0)=I$ to $\bar{U}_{A}(2 \pi)=e^{i 2 \pi / 3} I$, defined by a diagonal unitary matrix with nontrivial elements $e^{i \phi_{\alpha}}$ such that $\phi_{1}=2 \chi / 3+[2(\pi-\zeta) / 3] \Theta(\chi-\pi)$, $\phi_{2}=-2 \chi / 3$, and $\phi_{3}=-\left(\phi_{1}+\phi_{2}\right) ; \Theta(\chi)$ is the Heaviside function. For maximally entangled states, we have

$$
\langle\psi(0) \mid \psi(t)\rangle= \begin{cases}\frac{1}{3}\left[1+2 \cos \left(\frac{2 \chi}{3}\right)\right], & \chi \in[0, \pi] \\ \frac{1}{3}\left[1+2 \cos \left(\frac{2(\chi+\pi)}{3}\right)\right] e^{i \frac{2 \pi}{3}}, & \chi \in[\pi, 2 \pi] .\end{cases}
$$

Then, we see that the total phase vanishes in the first part of the evolution, while it takes the fractional value $2 \pi / 3$ in the second part. In addition, at $\chi=\pi$, when the phase changes discontinuously, the state $|\psi(t)\rangle$ becomes orthogonal to the initial state.

Both qutrit evolutions are represented in fig $2 \mathrm{a}$, where the overlap $\langle\psi(0) \mid \psi(t)\rangle$ is plotted in the complex plane for maximal concurrence. The first cyclic evolution from $P$ to $P^{\prime}$ is represented by the solid black line clearly showing that the overlap between the initial and the evolving quantum states never vanishes. On the other hand, the 

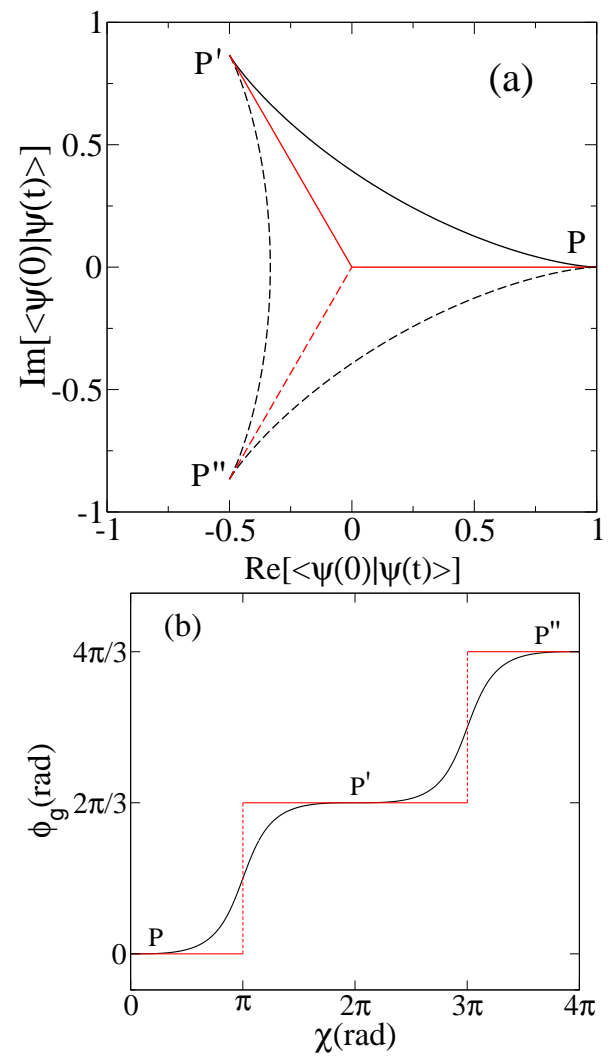

FIG. 2. (a) Complex plane representation of the quantum state overlap for a pair of qutrits with maximal concurrence. Two different time evolutions are considered. (b) The corresponding stepwise evolution of the geometric phase.

second evolution (red online) shows a path crossing the origin of the complex plane, where the evolving quantum state becomes orthogonal to the initial one. The dashed lines correspond to additional closed paths defining three vertices which evidence the fractional phase values. In fig $2 \mathrm{~b}$, we plot the associated geometric phase evolution, showing a stepwise behavior with two jumps between the fractional values $0,2 \pi / 3$, and $4 \pi / 3$. For the first evolution (black) smooth jumps occur, while for the second evolution (red online) they are discontinuous.

As a conclusion, in this letter we studied unitary local operations on a pair of qudits, showing that fractional phases naturally appear when cyclic evolutions are considered. These fractional values are related to different homotopy classes of closed paths in the two-qudit Hilbert space. The geometric phase has been calculated in terms of the I-concurrence introduced in ref. 13]. In the case of maximally entangled states, the fractional values originate solely from the geometric part of the phase evolution, since the dynamical part vanishes at all times.

The fractional phase of maximally entangled states is built in a stepwise evolution, where the phase jumps between discrete values in steps of $2 \pi / d$. For qubits this jump is strictly discontinuous, while for qutrits, it may be discontinuous or not, depending on the particular evolution considered. Due to its stepwise evolution, we expect the fractional phase acquired by maximally entangled qudits to be particularly robust against the influence of the environment. In order to produce a relevant change, any external noise would have to cause a large fluctuation, driving the two-qudit system through a phase step. Since the phase jump for qubits is strictly discontinuous, its robustness should be even more pronounced. These results can be important to proposals of quantum gates based on topological phases.

\section{ACKNOWLEDGEMENTS}

We are grateful to M. Sarandy, P. Milman and E. Sjöqvist for useful discussions. Funding was provided by Coordenação de Aperfeiçoamento de Pessoal de Nível Superior (CAPES), Fundação de Amparo à Pesquisa do Estado do Rio de Janeiro (FAPERJ-BR), and Instituto Nacional de Ciência e Tecnologia de Informação Quântica (INCT-CNPq).

[1] M. V. Berry, Proc. R. Soc. London A 392, 45 (1984).

[2] J. A. Jones, V. Vedral, A. Ekert, and G. Castagnoli, Nature (London) 403, 869 (2000).

[3] L.-M. Duan, J. I. Cirac, and P. Zoller, Science 292, 1695 (2001).

[4] S. Pancharatnam, Proc. Ind. Acad. Sci. 44, 247 (1956).

[5] S.J. van Enk, Opt. Comm. 102, 59 (1993).

[6] E. J. Galvez, P. R. Crawford, H. I. Sztul, M. J. Pysher, P. J. Haglin, and R. E. Williams, Phys. Rev. Lett.90, 203901 (2003).

[7] E. Sjöqvist, Phys. Rev. A62, 022109 (2000).

[8] B. Hessmo and E. Sjöqvist, Phys. Rev. A62, 062301 (2000).

[9] P. Milman, and R. Mosseri, Phys. Rev. Lett.90, 230403 (2003).

[10] P. Milman, Phys. Rev. A73, 062118 (2006).

[11] C. E. R. Souza, J. A. O. Huguenin, P. Milman, and A. Z. Khoury, Phys. Rev. Lett.99, 160401 (2007).

[12] J. Du, J. Zhu, M. Shi, X. Peng, and D. Suter, Phys. Rev. A76, 042121 (2007).

[13] P. Rungta, V. Buzek, C. M. Caves, M. Hillery and G. J. Milburn, Phys. Rev A64, 042315 (2001).

[14] N. Mukunda and R. Simon, Ann. Phys. 228, 205 (1993).

[15] W. S. Bae, Y.M. Cho and Sang-Woo Kim, Phys. Rev. D65, 025005 (2002).

[16] L. E. Oxman, JHEP 12089 (2008). 